\title{
Controlling precedence in sequential stimulus presentation with Euler tours
}

\author{
Jacob J.W. Bakermans ${ }^{1, *}$ and Timothy E.J. Behrens ${ }^{1,2,3}$
}

\begin{abstract}
It is important to control for stimulus history in experiments probing responses to and similarity between sequentially presented stimuli. We present a method for stimulus order randomisation that guarantees identical precedence across stimuli. Generating sequences through sampling Euler tours allows for perfectly uniform stimulus history. This deconfounds the stimulus history from the present stimulus and maximises sensitivity to stimulus history effects including repetition suppression.
\end{abstract}

\section{PROBLEM}

Many experimental paradigms in psychology and neuroscience present stimuli sequentially, meaning that past stimuli might influence the current neural response. Such stimulus history effects are interesting to measure as they may imply rich temporal processing in the brain [1][2], but they are also important to consider when trying to measure representational similarity via repetition suppression [3][4][5], or simply to deconfound the response to the current stimulus. It is therefore desirable to control experimentally the frequency with which different stimulus histories occur. Sequence position can be controlled for by randomising the order of stimulus presentation. However, randomisation by shuffling the presentation order will not fully control for stimulus history effects, as certain pairs of subsequent stimuli might occur more frequently than others. How can we randomise stimulus order with identical precedence across stimuli?

Methods for counterbalancing stimulus history [6] have focused on hemodynamic response estimation efficiency [7], orthogonality of stimulus and sequence position [8], or high hypothesis detection power [9], but not on sampling uniformity. Here we present a sequence randomisation method based on the Euler algorithm introduced by Kandel et al. [10] that uniformly samples sequences with balanced history of arbitrary length. We show extensions and provide a Python implementation for generating sequences under a range of experimental requirements. In principle, although we developed it independently, our method is highly similar to Aguirre et al. [9] apart from the sampling mechanism; in practice we hope the description and implementation provided here still prove useful to some.

\footnotetext{
${ }^{1}$ Wellcome Centre for Integrative Neuroimaging, University of Oxford, Oxford, OX3 9DU, UK

${ }^{2}$ Wellcome Centre for Human Neuroimaging, University College London, London, WC1N 3AR, UK

${ }^{3}$ Sainsbury Wellcome Centre for Neural Circuits and Behaviour, University College London, London W1T 4JG, UK

* jacob.bakermans@nden.ox.ac.uk
}

\section{SOLUTION}

Our goal is to generate a random sequence of stimuli that contains each pair of subsequent stimuli equally often. Fig 1 a shows an example of such a sequence $S$ for four stimuli. The first pair in $S$ is $1 \rightarrow 3$ (labelled A in Fig 1 1 ), followed by the pair $3 \rightarrow 4$ (B), then $4 \rightarrow 2$ (C), until all possible pairs have come along. In addition to equal stimulus pair count, $S$ also contains each individual stimulus equally often, except for one additional presentation of the first stimulus. In order to randomly generate these sequences, we will consider them as walks on a graph. We start by constructing a complete digraph $G$ with a vertex for each stimulus as in Fig 1 b. Each edge represents the transition from one stimulus to another. Sequence $S$ corresponds to a walk that starts at vertex (stimulus) 1 , follows the edge (transition) labelled A to vertex 3 , et cetera, until it arrives back at vertex 1 - after using each edge exactly once. It turns out that any walk that traverses each edge exactly once produces the kind of sequence we are looking for. Such a walk is called an Euler tour. Since each vertex of $G$ has equal indegree and outdegree, $G$ is Eulerian, and an Euler tour always exists. Our task boils down to sampling an Euler tour on $G$; Kandel et al. [10] invented the Euler algorithm that provably samples Euler tours randomly uniformly.

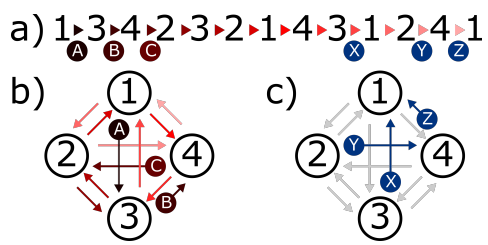

Fig. 1. a) Example sequence $S$ for four stimuli. Each pair is indicated by an arrow coloured by sequence position. b) Complete digraph $G$ for four stimuli with edges coloured to correspond to their position on the Euler tour of sequence $S$. The first three edges are labelled A, B, C in both the graph $G$ and sequence $S$. c) Sampled arborescence for generating sequence $S$, with edges in the arborescence in blue. The last transition from each vertex is taken from the arborescence, shown by the pairs labelled $\mathrm{X}, \mathrm{Y}, \mathrm{Z}$ in $S$.

The crucial step of the Euler algorithm is sampling an arborescence: a sub-set of the edges in $G$ so that there is exactly one path from each vertex to a root vertex (the first stimulus of the sequence). There are many arborescences for $G$; Fig 1k shows an example arborescence for root vertex 1. We generate an Euler tour by walking along $G$, picking an unused outgoing edge at random at each vertex - but we only take the edge that belongs to the arboresence at a vertex once no other unused edges remain. This way, the arboresence serves two purposes: it makes sure that we can 
complete the tour by returning to the initial vertex in the end, and it prevents us from getting stuck there before having used all other edges.

So how do we sample a random arborescence? The Euler algorithm constructs an arborescence using a backward random walk. Starting at the root vertex $v$ (stimulus 1 in Fig 1c), we randomly choose an incoming edge $e$ (e.g. edge $X$ in Fig 1 ), and transition in the opposite direction of $e$ to its source $v^{\prime}$ (stimulus 3 in Fig 1 F). If this is the first time $v^{\prime}$ (stimulus 3 ) is reached in the random walk, we add $e$ (edge $X)$ to the arborescence. Then we repeat the same procedure from $v^{\prime}$ (stimulus 3 ) to continue the random walk until all vertices have been covered. Kandel et al. [10] prove that the resulting arborescence is drawn from the uniform distribution over arborescences.

\section{EXTENSIONS}

By adapting the graph $G$ we can accommodate for various experimental requirements within the same algorithm. Here we list a number of such adaptations, further illustrated in Fig 2 .

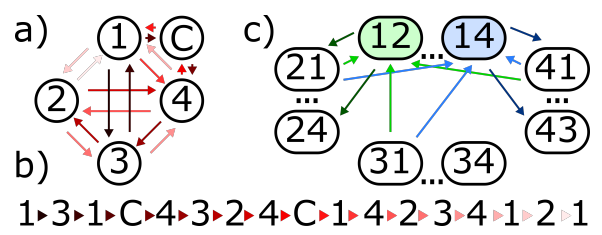

Fig. 2. Graph $G$ augmented with catch vertex C (a) with edges coloured along sampled Euler route (b) with catch trials. c) For controlling triplets instead of pairs, redefine vertices as pairs (e.g. $1-2 / 1-4$ in green/blue) and add edges between vertices that form triplets (light hue: incoming edges, dark hue: outgoing edges).

- Repeat stimuli. To include pairs consisting of identical stimuli, such as $1 \rightarrow 1$, add a loop for each vertex: an edge from the vertex back to itself.

- Repeat pairs. There are two ways to generate longer sequences with multiple repetitions of each stimulus pair: either duplicate edges between vertices, or generate multiple tours and stitch them together. The latter guarantees equal numbers of pairs in both halves of the resulting sequence.

- Add catch trials. Catch trials test the participant's attention but are not of (main) interest. Randomly inserting catch trials after generating a sequence would result in certain stimulus pairs being 'broken' by the catch trial. A better way to incorporate catch trials in the sequence is to add a catch vertex to $G$, and adding bidirectional edges until the desired catch frequency is achieved as in Fig 2 a. Indeed, the example sequence of Fig $2 \mathrm{p}$ contains all pairs of subsequent stimuli, despite the insertion of catch trials. If it is crucial that catch trials themselves precede each stimulus equally often, the catch vertex should be connected to all stimulus vertices.

- Control multi-step precedence. We can control not only for direct stimulus precedence, but for an arbitrary number of steps [10]. In case of two steps, we need each triplet of stimuli to occur equally often in the sequence instead of each pair. This is achieved by redefining $G$ so that vertices represent stimulus pairs (e.g. $1 \rightarrow 2,2 \rightarrow 4$ ) and only adding directed edges between pairs that form triplets (e.g. $1 \rightarrow 2$ and $2 \rightarrow 4$ form $1 \rightarrow 2 \rightarrow 4$ ) (Fig 2p).

- Induce non-uniform precedence. So far we have described procedures for generating sequences with uniform stimulus precedence. The method also allows for non-uniform precedence, with certain stimulus pairs occurring more often than others. Graph $G$ can be rewired to produce different pair frequencies, as long as it remains Eulerian: the indegree and outdegree need to be identical for each vertex.

\section{CODE}

A Python implementation is available at
https://github.com/jbakermans/
stimulus-history-euler-tours

\section{ACKNOWLEDGEMENTS}

We thank the following funding sources: EPSRC DPhil Scholarship to J.J.W.B.; Wellcome Principal Research Fellowship (219525/Z/19/Z), Wellcome Collaborator award (214314/Z/18/Z), and JS McDonnell Foundation award (JSMF220020372) to T.E.J.B.; the Wellcome Centre for Integrative Neuroimaging and Wellcome Centre for Human Neuroimaging are each supported by core funding from the Wellcome Trust (203139/Z/16/Z, 203147/Z/16/Z).

\section{REFERENCES}

[1] Akrami, Athena et al. "Posterior parietal cortex represents sensory history and mediates its effects on behaviour." Nature vol. 554,7692 (2018): 368-372. doi:10.1038/nature25510

[2] Fritsche, Matthias, Samuel G. Solomon, and Floris P. de Lange. "Brief stimuli cast a long-term trace in visual cortex." bioRxiv (2021).

[3] Gross, Charles G., et al. "Single-unit activity in temporal association cortex of the monkey." Journal of Neurophysiology 30.4 (1967): 833843

[4] Tootell, Roger BH, et al. "Visual motion aftereffect in human cortical area MT revealed by functional magnetic resonance imaging." Nature 375.6527 (1995): 139-141.

[5] Kourtzi, Zoe, and Nancy Kanwisher. "Representation of perceived object shape by the human lateral occipital complex." Science 293.5534 (2001): 1506-1509.

[6] Aguirre, Geoffrey Karl. "Continuous carry-over designs for fMRI." Neuroimage 35.4 (2007): 1480-1494.

[7] Buračas, Giedrius T., and Geoffrey M. Boynton. "Efficient design of event-related fMRI experiments using M-sequences." Neuroimage 16.3 (2002): 801-813.

[8] Nonyane, B. A. S., and C. M. Theobald. "Design sequences for sensory studies: Achieving balance for carry-over and position effects." British Journal of Mathematical and Statistical Psychology 60.2 (2007): 339349 .

[9] Aguirre, Geoffrey Karl, Marcelo Gomes Mattar, and Lucía MagisWeinberg. "de Bruijn cycles for neural decoding." NeuroImage 56.3 (2011): 1293-1300.

[10] Kandel, D., et al. "Shuffling biological sequences." Discrete Applied Mathematics 71.1-3 (1996): 171-185. 